\title{
Clinical Significance of Serum Galectin-1 and Its Tissue Immunohistochemical Expression in Serous Ovarian Carcinoma Patients
}

\author{
Mai Mohamed Abdelwahab ${ }^{1 *}$, Huda Fathy Ebian2, Taiseer Refat Ibrahim1, \\ Mohammad Samir Badr'3, Mohamed El-Bakry Lashin ${ }^{3}$, Mahmoud Abdou Yassin", \\ Adel Mohamed Ismail ${ }^{5}$, Ahmed Ali Obaya ${ }^{6}$
}

\author{
${ }^{1}$ Department of Pathology, Faculty of Medicine, Zagazig University, Zagazig, Egypt \\ ${ }^{2}$ Department of Clinical Pathology, Faculty of Medicine, Zagazig University, Zagazig, Egypt \\ ${ }^{3}$ Department of Obstetrics and Gynecology, Faculty of Medicine, Zagazig University, Zagazig, Egypt \\ ${ }^{4}$ Department of General Surgery, Faculty of Medicine, Zagazig University, Zagazig, Egypt \\ ${ }^{5}$ Department of Surgical Oncology, Ismailia Teaching Oncology Hospital, Ismailia, Egypt \\ ${ }^{6}$ Department of Clinical Oncology, Faculty of Medicine, Zagazig University, Zagazig, Egypt \\ Email: *maiabdelwahab@ymail.com
}

How to cite this paper: Abdelwahab, M.M., Ebian, H.F., Ibrahim, T.R., Badr, M.S., Lashin, M.E.-B., Yassin, M.A., Ismail, A.M. and Obaya, A.A. (2019) Clinical Significance of Serum Galectin-1 and Its Tissue Immunohistochemical Expression in Serous Ovarian Carcinoma Patien. Open Journal of Obstetrics and Gynecology, 9, 937-953.

https://doi.org/10.4236/ojog.2019.97091

Received: June 16, 2019

Accepted: July 2, 2019

Published: July 5, 2019

Copyright $\odot 2019$ by author(s) and Scientific Research Publishing Inc. This work is licensed under the Creative Commons Attribution International License (CC BY 4.0).

http://creativecommons.org/licenses/by/4.0/

(c) (i) Open Access

\begin{abstract}
Objectives: Serous ovarian carcinoma (SOC) is the commonest ovarian carcinoma type with poor prognosis due to early metastasis and first presentation with advanced stage. In this work, we investigated serum level of Galactin-1 (Gal-1) and its tissue immunohistochemical expression in SOC patients at different stages trying to find out its significance as a diagnostic and prognostic marker. Patients and methods: The study included 95 females I-Control group: Twenty five healthy females; II-Patients group: Seventy females diagnosed as SOC at different stages; Stage I: 8 cases, Stage II: 12 cases, Stage III: 32 cases and Stage VI: 18 cases. Serum Galectin- 1 and CA-125 were measured by ELIZA and tissue Galectin-1 was assessed by immunohistochemistry. All patients were followed for up to 3 years after surgery. Results: Serum Gal-1 and CA-125 levels were significantly higehr in SOC patients compared to controls $(\mathrm{p}<0.001)$. We found a direct positive statistically significant correlation between serum Gal-1 and CA125 levels ( $\mathrm{p}<0.001$ ). Serum Gal-1 at cut off value $>135 \mathrm{ng} / \mathrm{ml}$ was superior to CA-125 a cut off value $>49 \mathrm{u} / \mathrm{ml}$ with sensitivity, specificity of $100 \%$, vs 88.57 , $96 \%$ for CA- 125 . Serum Gal-1 was significantly associated with tumor stage $(\mathrm{p}<0.001)$. Immunohistochemistry showed that patients with strong Gal-1 expression had higher serum level $(p=0.002)$. Stromal and tumor Gal-1 expression were significantly correlated with tumor grade $(\mathrm{p}<0.001)$ and stage $(\mathrm{p}=0.001)$. Serum Gal-1, CA-125 and IHC Gal-1 expression were associated with poor sur-
\end{abstract}


vival ( $\mathrm{p}<0.001, \mathrm{p}=0.009$ and $\mathrm{p}=0.002$ ) respectively. Conclusion: Serum Gal-1 and its tissue IHC expression are useful diagnostic and prognostic markers for SOC patients.

\section{Keywords}

Serous Ovarian Carcinoma, Serum, Immunohistochemistry, Galectin-1, Overall Survival

\section{Introduction}

Ovarian carcinoma (OC) is the most prevalent gynecologic malignancy. Serous ovarian carcinoma (SOC) is the most common subtype. That is grouped into 2 types based on histologic characteristics (high-grade and low-grade) [1].

The standard first-line treatment for these tumors is debulking surgery followed by chemotherapy. However, the 5-year survival rate is less than $50 \%$ due to local invasion, early metastasis and the first presentation with advanced disease stage [2].

The outcome in these patients depends on stage at diagnosis, extent of residual disease after surgery and histological subtype [3].

High grade serous ovarian cancer (HGSC) is considered the most lethal and frequent type of epithelial ovarian cancer (EOC). It has poor long term prognosis due to a combination of factors: late detection, great metastatic potential and resistance to available therapeutic drugs [4].

Various studies aimed to introduce new biological prognostic markers in ovarian carcinoma. Estrogen receptor promoter methylation can be used to predict overall survival in low grade tumors [5]. However, till today, no reliable biological marker is generally accepted [3]. The improvement of biological prognostic markers is necessary for specification of anti-cancer therapy [6].

The most clinically valuable ovarian carcinoma biomarker, CA125, has been used to evaluate therapeutic response and check recurrence of EOC. However, it lacks both sensitivity and specificity. Elevated CA125 levels in multiple gynecological conditions decreased its specificity; also, a high proportion of epithelial ovarian cancers do not express CA125 [7].

Galectins constitute a gene family of widely distributed B-galactoside-binding glycoproteins with high affinity for $\beta$-galactoside. It is expressed in many tumor types such as astrocytoma, melanoma, thyroid, colon, bladder, and ovarian cancers [8].

Galectin-1 is involved in many physiological and pathological process including cell-cell and cell-matrix interactions, cell growth, inflammatory reaction, immune regulation, cell differention and tumor progress [9].

Several studies suggest that dysregulation of galectin-1 has a link to invasion and metastasis, formation of cancer cells which will promote growth of tumors, angiogenesis and keep the cells away from being destroyed by the immune re- 
sponse of the host [10].

It was found that galectin-1 is increased in cancer cells as reported in thyroid carcinoma by Chiariotti et al., 1995 [11] and by Xu et al., 1995 [12]. Also, increased galectin-1 expression presented with breast cancer and ovarian cancer [13].

Gal-1 was detected in neighboring cancer associated fibroblasts and cancer stroma of the primary prostate cancer by Van den Brule et al., 2001 [14], though Galectin-1 is strongly expressed in ovarian cancer [15]. However, its potential usefulness as a diagnostic or prognostic marker remains unclear.

In this work, we investigated serum level of Galactin-1 and its cellular expression in serous epithelial ovarian cancer in different stages trying to find out its significance as a diagnostic and prognostic marker.

\section{Patients and Methods}

\section{Patients}

This is a cross sectional study, conducted in Gynecology and Obstetrics, pathology, clinical pathology and clinical oncology departments, Zagazig University Hospitals between March 2016 and April 2019. Ethical approval was granted by Zagazig faculty of Medicine Research and Ethics Committee prior to conducting the study. Full clinical data together with informed consent were obtained from all subjects prior to sample collection.

Surgical assessment was used to decide the clinical stages and metastases presence according to the FIGO classification 2014. Histopathological evaluation was carried out to determine cancer types and the grades.

The study included 95 females that were divided into I-Control group: Twenty five healthy females.

II-Patients group: Seventy females who were diagnosed to have serous ovarian carcinoma (SOC) at different stages: Stage I: 8 cases, Stage II: 12 cases, Stage III: 32 cases and Stage VI: 18 cases. Eighteen cases were low grade, while 52 cases were high grade. We excluded any patient who had previous chemo or radiotherapy and females with benign or borderline tumors.

All patients were followed for up to 3 years after surgery.

\section{Methods}

\subsection{Serum, ELIZA}

About $2 \mathrm{ml}$ of blood samples were collected from patients and control under aseptic conditions. Samples were delivered to a plain tube and allowed to clot. Then serum was aliquoted in clean vials and stored frozen at $-20^{\circ} \mathrm{C}$. Enzyme immunoassay technique was used for measurement of serum Gal-1 concentration-ELISA method (eBioscince, Vienna, Austria) [16].

Galactin-1 level was measured using Quantikine ${ }^{\oplus}$ ELISA Human Galectin-1 Immunoassay kit (ELISA method (eBioscince, Vienna, Austria) Catalog Number DGAL10) and an ELISA plate reader b (Tecan-Austria GM bit.8 Gro dig. Aus- 
tria, following the manuel of the procedure. The sample kept at RT 8-25C). Determine optical density (OD) value of each well at microplate reader $(450 \mathrm{~nm})$. The minimum detectable dose (MDD) of human Galectin-1 ranged from 0.008 $0.129 \mathrm{ng} / \mathrm{mL}$. The mean MDD was $0.022 \mathrm{ng} / \mathrm{mL}$.

Serum levels of CA125 were determined by chemiluminescent enzyme immunoassay Cobas 6000 e601 Tokyo Japan 7Z81), considering CA125 values higher than $35 \mathrm{U} / \mathrm{mL}$ as abnormal.

\subsection{Immunohistochemistry}

Five $\mu \mathrm{m}$ thick tissue sections were deparaffinized in xylene and then dehydrated in graded ethanol. $0.3 \%$ hydrogen peroxide in methanol was used to block the endogenous peroxidase activity for $15 \mathrm{~min}$. Then, rinsing in phosphate buffered saline (PBS) at a $\mathrm{pH}$ of 7.2,10\% bovine serum (Wako, Osaka, Japan) was applied for $20 \mathrm{~min}$ in order to block any non-specific reactions. Sections were incubated after that overnight with the primary antibody at $4^{\circ} \mathrm{C}$ : anti-galectin-1 (Santa Cruz, CA, USA). Then rinsed in PBS, incubated with a biotin-conjugated secondary antibody followed by incubation using the streptavidin-biotin system for about $30 \mathrm{~min}$ at room temperature. The peroxidase reaction was visualized by incubating the sections with diaminobenzidine tetra-hydrochloride (DAB). The sections were counterstained with hematoxylin, then rinsed, and mounted. Macrophages and endothelial cells served as positive internal control. Negative controls were obtained by replacing the primary antibody with non-immune serum.

\subsection{Assessment of Immunohistochemistry}

For assessment of galectin-1 immunohistochemical expression, each case was evaluated for the intensity of staining and extent. Ten high-power fields were selected randomly, and more than 1000 cells were counted for each section. The intensity of staining was assessed as follow: 0 , no staining; $+1=$ mild staining; +2 $=$ moderate staining; $+3=$ intense staining. The extent of staining was graded as follows: $0=$ no staining of cells in any fields; $+1<30 \%$ of tissue stained positive; $+2=$ between $30 \%$ and $60 \%$ of tissue are stained positive; $+3 \geq 60 \%$ of tissue are stained positive. A total score obtained by summation of (Intensity + extent). So, ranged from 0 to maximum, 6 . A combined staining score of $\leq 2$ was considered being a negative staining; a score of three was considered a weak staining; while a score between 4 and 6 was considered to be a strong staining [17].

\subsection{Statistical Analysis}

Continuous variables were expressed as mean \pm SD \& median, while the categorical variables were expressed as a number (percentage). Continuous variables were checked for normality by using Shapiro-Wilk test. Mann-Whitney U test was used to compare between two groups of non-normally distributed data. Kruskal Wallis $\mathrm{H}$ test was used to compare between more than two groups of non-normally distributed data. Percent of categorical variables were compared 
using Pearson's Chi-square test or Fisher's exact test when was appropriate. Trend of change in distribution of relative frequencies between ordinal data were compared using Chi-square test for trend. Strength of relationship between age, CA125 and Gal-1 were determined by computing Spearman's rank correlation coefficient with $(+)$ sign was indicator for direct relationship \& (-) sign was indication for inverse relationship, values near to 1 was indicator for strong relationship \& values near 0 was indicator for weak relationship. Receiver operating characteristic (ROC) curve analysis was used to identify optimal cut-off values of CA125 and Gal-1 with maximum sensitivity and specificity for diagnosis of ovarian carcinoma. Area Under Curve (AUROC) was also calculated, criteria to qualify for AUC were as follows: $0.90-1=$ excellent, $0.80-0.90=$ good, $0.70-$ $0.80=$ fair; $0.60-0.70=$ poor; and $0.50-0.6=$ fail. The optimal cutoff point was established at point of maximum accuracy. Overall Survival (OS) was considered from time of diagnosis to death or the most recent follow-up contact (censored). Stratification of OS was done according all clinicopathological and IHC staining. These time-to-event distributions were anticipated using the method of Kaplan-Meier plot, and compared using two-sided exact log-rank test. All tests were two sided. A p-value $<0.05$ was considered significant. All statistics were performed using SPSS 22.0 for windows (SPSS Inc., Chicago, IL, USA) and MedCalc windows (MedCalc Software bvba 18, Ostend, Belgium).

\section{Results}

\subsection{Clinicopathological Results}

The present study included 70 patients with SOC and 25 healthy controls. The age range of the studied patients was $27-62$ years, mean age was $50.24 \pm 9.58$ years, and median age was 52.5 years. $18 / 70$ of cases were low grade $(27.7 \%)$ and 52 cases were high grade (74.3\%). All were studied for of serum CA 125 and Gal-1 and for tissue Gal-1 immunohistochemical expression. CA125 level ranged from $(69-2008) \mathrm{u} / \mathrm{ml}$ with mean $1071.31 \pm 666.91 \mathrm{u} / \mathrm{ml}$; Serum Gal-1 level ranged from $(32-763) \mathrm{ng} / \mathrm{ml}$ with mean $334.84 \pm 187.67 \mathrm{ng} / \mathrm{ml}$. $68.6 \%$ showed strong IHC expression in cancer stromal cells, and $65.7 \%$ showed strong expression in tumor cells. Death rate was $22.9 \%$ during the follow up period. The clinicopathological data of our studied cases are summarized in Table 1.

\subsection{Serum CA 125 and Galectin-1 Levels from Normal Controls and SOC Patients}

The mean value of CA-125 in sera of 25 healthy controls was $(22.28 \pm 8.98)$, while in SOC patients; the mean was $(1071.31 \pm 666.91) \mathrm{u} / \mathrm{ml}$.

The mean serum Gal-1 level in controls was $86.36 \pm 30.39 \mathrm{ng} / \mathrm{ml}$; while in SOC patients , the mean was $334.84 \pm 187.67 \mathrm{ng} / \mathrm{ml}$.

Threre was a statistcally significant difference between controls and SOC patients as regards to serum CA 125 and serum Gal-1 levels (p < 0.001) (Table 2). 
Table 1. Clinicopathological features, immunohistochemical staining and outcome among 70 ovarian carcinoma patients.

\begin{tabular}{|c|c|c|}
\hline \multirow{2}{*}{ Characteristics } & \multicolumn{2}{|c|}{ All $(\mathrm{N}=70)$} \\
\hline & No. & $\%$ \\
\hline \multicolumn{3}{|l|}{ Age (years) } \\
\hline Mean \pm SD & \multicolumn{2}{|c|}{$50.24 \pm 9.58$} \\
\hline Median (Range) & \multicolumn{2}{|c|}{$52.50(27-62)$} \\
\hline \multicolumn{3}{|l|}{ Grade } \\
\hline Low & 18 & $27.7 \%$ \\
\hline High & 52 & $74.3 \%$ \\
\hline \multicolumn{3}{|l|}{$\underline{\mathrm{LN}}$} \\
\hline Negative & 22 & $31.4 \%$ \\
\hline Positive & 48 & $68.6 \%$ \\
\hline \multicolumn{3}{|l|}{$\underline{\text { Stage }}$} \\
\hline Stage I & 8 & $11.4 \%$ \\
\hline Stage II & 12 & $17.1 \%$ \\
\hline Stage III & 32 & $45.7 \%$ \\
\hline Stage IV & 18 & $25.7 \%$ \\
\hline \multicolumn{3}{|l|}{$\underline{\operatorname{CA} 125(\mathrm{u} / \mathrm{ml})}$} \\
\hline Mean \pm SD & \multicolumn{2}{|c|}{$1071.31 \pm 666.91$} \\
\hline Median (Range) & \multicolumn{2}{|c|}{$1238.50(69-2008)$} \\
\hline \multicolumn{3}{|l|}{ Gal-1 (ng/ml) } \\
\hline Mean \pm SD & \multicolumn{2}{|c|}{$334.84 \pm 187.67$} \\
\hline Median (Range) & \multicolumn{2}{|c|}{$277(32-763)$} \\
\hline \multicolumn{3}{|l|}{$\underline{\text { Gal-1 }}$} \\
\hline Weak & 22 & $31.4 \%$ \\
\hline Strong & 48 & $68.6 \%$ \\
\hline \multicolumn{3}{|l|}{$\underline{\text { Gal-1 tumor cells }}$} \\
\hline Weak & 24 & $34.3 \%$ \\
\hline Strong & 46 & $65.7 \%$ \\
\hline \multicolumn{3}{|c|}{ Follow-up duration (months) } \\
\hline Mean \pm SD & \multicolumn{2}{|c|}{$15.45 \pm 8.45$} \\
\hline Median (Range) & \multicolumn{2}{|c|}{$14(3-36)$} \\
\hline \multicolumn{3}{|l|}{ Outcome } \\
\hline Alive & 54 & $77.1 \%$ \\
\hline Died & 16 & $22.9 \%$ \\
\hline
\end{tabular}

Categorical variables were expressed as number (percentage). Continuous variables were expressed as mean \pm SD \& median (range). 
Table 2. Comparison between ovarian carcinoma cases and control regarding CA125 and serum Gal-1.

\begin{tabular}{cccc}
\hline & Control $(\mathrm{N}=25)$ & $\begin{array}{c}\text { Ovarian carcinoma cases } \\
(\mathrm{N}=70)\end{array}$ & p-value $\neq$ \\
\hline CA125 $(\mathrm{u} / \mathrm{ml})$ & & & \\
Mean $\pm \mathrm{SD}$ & $22.28 \pm 8.98$ & $1071.31 \pm 666.91$ & $<0.001$ \\
Median (Range) & $21(8-49)$ & $1238.50(69-2008)$ & \\
Gal-1 (ng/ml) & & & \\
Mean \pm SD & $86.36 \pm 30.39$ & $334.84 \pm 187.67$ & $<0.001$ \\
Median (Range) & $84(31-201)$ & $277(32-763)$ & \\
\hline
\end{tabular}

Continuous variables were expressed as mean $\pm \mathrm{SD}$ \& median (range). $\bullet$ Mann Whitney $\mathrm{U}$ test; $\mathrm{p}<0.05$ is significant.

\subsection{Diagnostic Performance of CA125 and Serum Gal-1 in Diagnosis of SOC: ROC Curve Analysis}

For diagnosis of SOC, the cut off value of Gal-1 $>135 \mathrm{ng} / \mathrm{ml}$ was found to have sensitivity of $100 \%$, specificity of $100 \%$, with positive and negative predictive values $100 \%$.

While for CA 125 , a cut off value $>49 \mathrm{u} / \mathrm{ml}$, have sensitivity $88.57 \%$, specificity of $96 \%$, positive predictive value $98.4 \%$ and negative predictive value $75 \%$.

The overall accuracy for Gal-1 was $100 \%$ and for CA125 was $90.5 \%$ in diagnosis of SOC (Table 3).

\subsection{Correlation between Galectin-1 and CA125 Levels}

Using Spearman's rank correlation coefficient; we found a direct positive statistically significant correlation between mean value of serum Gal-1 levels and CA125 levels ( $\mathrm{p}<0.001)$ (Table 4).

\subsection{Relation between Serum Gal-1 and Stage among SOC Patients}

We found a statistically significant association between serum Gal-1 level and tumor stage $\mathrm{p}<0.001$ (Table 5).

\subsection{Immunohistochemical Expression of Gal-1}

After immunohistochemical staining, high grade tumors showed stronger Gal-1 expression in pei-tumral stromal cells as well as in tumor cells; $82.7 \%$ and $84.6 \%$ respectively, and there was statistically significant difference in relation to those with weak expression $(\mathrm{p}<0.001)$.

Also, Gal-1 IHC expression was progressively frequent in patients with SOC as the stage of the disease progress both in stromal cells and tumor cells. Strong Gal-1 expression was present in $25 \%, 66.7 \%, 86.8 \%$ and $88.9 \%$ in stage I, II, III, IV respectively regarding stromal cells, while it was present in $37.5 \%, 50 \%$, $86.8 \%$ and $83.3 \%$ in tumor cells in stage I, II, III, IV respectively.; with statistically significant difference $(\mathrm{p}=0.001)$ compared with weak Gal-1 expression. 
Table 3. Diagnostic performance of CA125 and serum Gal-1 in diagnosis of ovarian carcinoma: ROC curve analysis.

\begin{tabular}{|c|c|c|c|c|c|c|c|}
\hline $\begin{array}{l}\text { Cut-off } \\
\text { values }\end{array}$ & $\begin{array}{c}\mathrm{SN} \% \\
(95 \% \mathrm{CI})\end{array}$ & $\begin{array}{c}\mathrm{SP} \% \\
(95 \% \mathrm{CI})\end{array}$ & $\begin{array}{c}\text { PPV \% } \\
\text { (95\% CI) }\end{array}$ & $\begin{array}{l}\text { NPV \% } \\
(95 \% \text { CI) }\end{array}$ & $\begin{array}{l}\text { Accuracy } \\
(95 \% \text { CI })\end{array}$ & $\begin{array}{l}\text { AUROC } \\
(95 \% \text { CI })\end{array}$ & $\mathrm{p}$-value \\
\hline $\begin{array}{c}\text { Gal-1 } \\
>135 \mathrm{ng} / \mathrm{ml}\end{array}$ & $\begin{array}{c}100 \% \\
(94.9-100)\end{array}$ & $\begin{array}{c}100 \% \\
(86.3-100)\end{array}$ & $\begin{array}{c}100 \% \\
(94.9-100)\end{array}$ & $\begin{array}{c}100 \% \\
(86.3-100)\end{array}$ & $\begin{array}{c}100 \% \\
(92.6-100)\end{array}$ & $\begin{array}{c}1.000 \\
(0.962-1.000)\end{array}$ & $<0.001$ \\
\hline $\begin{array}{c}\text { CA125 } \\
>49 \mathrm{u} / \mathrm{ml}\end{array}$ & $\begin{array}{c}88.57 \% \\
(78.7-94.9)\end{array}$ & $\begin{array}{c}96 \% \\
(79.6-99.9)\end{array}$ & $\begin{array}{c}98.4 \% \\
(91.4-100)\end{array}$ & $\begin{array}{c}75 \% \\
(56.6-88.5)\end{array}$ & $\begin{array}{c}90.5 \% \\
(78.9-96.2)\end{array}$ & $\begin{array}{c}0.907 \\
(0.829-0.957)\end{array}$ & $<0.001$ \\
\hline
\end{tabular}

Table 4. Correlation between CA125 and serum Gal-1.

\begin{tabular}{ccccc}
\hline & \multicolumn{3}{c}{ CA125 $(\mathrm{u} / \mathrm{ml})$} & \multicolumn{2}{c}{ Gal-1 $(\mathrm{ng} / \mathrm{ml})$} \\
\cline { 2 - 5 } & $\mathrm{r}$ & $\mathrm{p}$-value & $\mathrm{r}$ & $\mathrm{p}$-value \\
\hline CA125 $(\mathrm{u} / \mathrm{ml})$ & --- & --- & +0.640 & $<0.001$ \\
Gal-1 $(\mathrm{ng} / \mathrm{ml})$ & +0.640 & $<0.001$ & $-\cdots--$ & $\cdots$ \\
\hline
\end{tabular}

r: Spearman's rank correleation coefficient; p-value $<0.05$ is significant.

Table 5. Relation between serum Gal-1 and stage among 70 ovarian carcinoma patients.

\begin{tabular}{cccccc}
\hline & & & Gal-1 (ng/ml) & p-value $\neq$ \\
\cline { 3 - 4 } Stage & $\mathrm{N}$ & Mean $\pm \mathrm{SD}$ & Median & (Range) & $(32-165)$ \\
Stage I & 8 & $89.87 \pm 42.82$ & 76.50 & $(35-283)$ & $<0.001$ \\
Stage II & 12 & $236.16 \pm 77.05$ & 273.50 & $(95-386)$ & $(476-763)$ \\
Stage III & 32 & $275.15 \pm 64.40$ & 273 & 644 & \\
Stage IV & 18 & $615.61 \pm 74.25$ &
\end{tabular}

Continuous variables were expressed as mean $\pm \mathrm{SD} \&$ median (range). $\bullet$ Kruskal Wallis $\mathrm{H}$ test; $\mathrm{p}<0.05$ is significant.

We found that patients with stronger IHC expression of Gal-1 had significantly higher mean serum level of Gal-1 compared to those with weak expression $(\mathrm{p}=0.002)$.

There was a statistically significant difference between stromal and tumor Gal-1 strong and weak expression $(p<0.001)$ (Table 6, Figure 1).

\subsection{Survival Analysis Results}

The mean values of serum Gal-1 level was statistically significantly higher in dead compared to survivors ( $465.05 \pm 175.31 \mathrm{ng} / \mathrm{ml}$ vs $296.25 \pm 174.70 \mathrm{ng} / \mathrm{ml})(\mathrm{p}$ $<0.001$ ). Also, mean values of serum CA 125 levels was significantly higher in dead than survivors $(\mathrm{p}=0.009)$.

By IHC, stromal strong Gal-1 expression was significantly associated with poor survival compared to weak expression $(\mathrm{p}=0.002)$, while no statistically significant difference was found regarding tumor Gal-1 expression between survivors and non-survivors $(\mathrm{p}=0.136)$.

After follow up period of 3 years, the mean overall survival period was 28.2 months, with 1 year OS rate $83.2 \%$, 2-years OS rate $67.3 \%$ and 3 -year OS rate $61.3 \%$. 
Table 6. Relation between clinicopathological features and immunohistochemical staining among 70 ovarian carcinoma patients.

\begin{tabular}{|c|c|c|c|c|c|c|c|c|c|c|c|c|}
\hline \multirow{3}{*}{ Characteristics } & \multirow{2}{*}{\multicolumn{2}{|c|}{$\begin{array}{c}\text { All } \\
(\mathrm{N}=70)\end{array}$}} & \multicolumn{4}{|c|}{ Gal-1 } & \multirow{3}{*}{$\mathrm{p}$-value } & \multicolumn{4}{|c|}{ Gal-1 tumor cells } & \multirow{3}{*}{$\mathrm{p}$-value } \\
\hline & & & \multicolumn{2}{|c|}{$\begin{array}{c}\text { Weak } \\
(\mathrm{N}=22)\end{array}$} & \multicolumn{2}{|c|}{$\begin{array}{c}\text { Strong } \\
(\mathrm{N}=48)\end{array}$} & & \multicolumn{2}{|c|}{$\begin{array}{c}\text { Weak } \\
(\mathrm{N}=24)\end{array}$} & \multicolumn{2}{|c|}{$\begin{array}{c}\text { Strong } \\
(\mathrm{N}=46)\end{array}$} & \\
\hline & No. & $\%$ & No. & $\%$ & No. & $\%$ & & No. & $\%$ & No. & $\%$ & \\
\hline \multicolumn{13}{|l|}{ Age (years) } \\
\hline Mean \pm SD & \multicolumn{2}{|c|}{$50.24 \pm 9.58$} & \multicolumn{2}{|c|}{$49.13 \pm 9.13$} & \multicolumn{2}{|c|}{$50.75 \pm 9.84$} & \multirow[b]{2}{*}{$0.265^{\bullet}$} & \multicolumn{2}{|c|}{$50.50 \pm 9.82$} & \multicolumn{2}{|c|}{$50.10 \pm 9.56$} & \multirow[b]{2}{*}{$0.848 \bullet$} \\
\hline $\begin{array}{l}\text { Median } \\
\text { (Range) }\end{array}$ & \multicolumn{2}{|c|}{$\begin{array}{c}52.50 \\
(27-62)\end{array}$} & \multicolumn{2}{|c|}{$\begin{array}{c}50 \\
(27-61)\end{array}$} & \multicolumn{2}{|c|}{$\begin{array}{c}54 \\
(27-62)\end{array}$} & & \multicolumn{2}{|c|}{$\begin{array}{c}53.50 \\
(27-61)\end{array}$} & \multicolumn{2}{|c|}{$\begin{array}{c}52 \\
(27-62)\end{array}$} & \\
\hline \multicolumn{13}{|l|}{$\underline{\text { Grade }}$} \\
\hline $\begin{array}{l}\text { Low } \\
\text { High }\end{array}$ & $\begin{array}{l}18 \\
52\end{array}$ & $\begin{array}{l}27.7 \% \\
74.3 \%\end{array}$ & $\begin{array}{c}13 \\
9\end{array}$ & $\begin{array}{l}72.2 \% \\
17.3 \%\end{array}$ & $\begin{array}{c}5 \\
43\end{array}$ & $\begin{array}{l}27.8 \% \\
82.7 \%\end{array}$ & $<0.001 \ddagger$ & $\begin{array}{l}16 \\
8\end{array}$ & $\begin{array}{l}88.9 \% \\
15.4 \%\end{array}$ & $\begin{array}{c}2 \\
44\end{array}$ & $\begin{array}{l}11.1 \% \\
84.6 \%\end{array}$ & $<0.001 \ddagger$ \\
\hline \multicolumn{13}{|l|}{$\underline{\mathrm{LN}}$} \\
\hline $\begin{array}{l}\text { Negative } \\
\text { Positive }\end{array}$ & $\begin{array}{l}22 \\
48\end{array}$ & $\begin{array}{l}31.4 \% \\
68.6 \%\end{array}$ & $\begin{array}{l}10 \\
12\end{array}$ & $\begin{array}{c}45.5 \% \\
25 \%\end{array}$ & $\begin{array}{l}12 \\
36\end{array}$ & $\begin{array}{c}54.5 \% \\
75 \%\end{array}$ & $0.087 \ddagger$ & $\begin{array}{l}11 \\
13\end{array}$ & $\begin{array}{r}50 \% \\
27.1 \%\end{array}$ & $\begin{array}{l}11 \\
35\end{array}$ & $\begin{array}{r}50 \% \\
72.9 \%\end{array}$ & $0.061 \ddagger$ \\
\hline \multicolumn{13}{|l|}{$\underline{\text { Stage }}$} \\
\hline Stage I & 8 & $11.4 \%$ & 6 & $75 \%$ & 2 & $25 \%$ & & 5 & $62.5 \%$ & 3 & $37.5 \%$ & \\
\hline $\begin{array}{l}\text { Stage II } \\
\text { Stage III }\end{array}$ & $\begin{array}{l}12 \\
32\end{array}$ & $\begin{array}{l}17.1 \% \\
45.7 \%\end{array}$ & $\begin{array}{c}4 \\
10\end{array}$ & $\begin{array}{l}33.3 \% \\
31.2 \%\end{array}$ & $\begin{array}{c}8 \\
22\end{array}$ & $\begin{array}{l}66.7 \% \\
68.8 \%\end{array}$ & $0.003 \S$ & $\begin{array}{c}6 \\
10\end{array}$ & $\begin{array}{c}50 \% \\
31.2 \%\end{array}$ & $\begin{array}{c}6 \\
22\end{array}$ & $\begin{array}{l}50 \% \\
68.8 \%\end{array}$ & $0.010 \$$ \\
\hline Stage IV & 18 & $25.7 \%$ & 2 & $11.1 \%$ & 16 & $88.9 \%$ & & 3 & $16.7 \%$ & 15 & $83.3 \%$ & \\
\hline CA125 (u/ml) & & & & & & & & & & & & \\
\hline Mean \pm SD & 1071.3 & \pm 666.91 & 806.77 & $=641.61$ & 1192. & \pm 649.05 & & 762.1 & 643.79 & 132.6 & \pm 626.57 & \\
\hline $\begin{array}{l}\text { Median } \\
\text { (Range) }\end{array}$ & & $\begin{array}{l}.50 \\
2008)\end{array}$ & $\begin{array}{r}12 \\
(69\end{array}$ & $\begin{array}{l}6.50 \\
1965)\end{array}$ & & $\begin{array}{l}46 \\
2008)\end{array}$ & $0.025^{\bullet}$ & & $\begin{array}{l}5 \\
2008)\end{array}$ & & $\begin{array}{l}8.50 \\
2005)\end{array}$ & $0.010^{\bullet}$ \\
\hline$\underline{\text { Gal-1 }(\mathrm{ng} / \mathrm{ml})}$ & & & & & & & & & & & & \\
\hline Mean \pm SD & 334.8 & 187.67 & 210.04 & $=135.00$ & 392.0 & 181.47 & & 253. & 171.94 & 377.3 & +183.07 & \\
\hline $\begin{array}{l}\text { Median } \\
\text { (Range) }\end{array}$ & & $\begin{array}{l}7 \\
763)\end{array}$ & & $\begin{array}{l}.50 \\
598)\end{array}$ & & $\begin{array}{l}32 \\
763)\end{array}$ & $<0.001 \bullet$ & & $\begin{array}{l}3 \\
651)\end{array}$ & & $\begin{array}{l}82 \\
763)\end{array}$ & $0.002 \bullet$ \\
\hline$\underline{\text { Gal-1 }}$ & & & & & & & & & & & & \\
\hline Weak & 22 & $31.4 \%$ & & & & & & 15 & $68.2 \%$ & 7 & $31.8 \%$ & \\
\hline Strong & 48 & $68.6 \%$ & & & & & & 7 & $18.8 \%$ & 39 & $81.2 \%$ & $=0.001 \mathrm{~T}$ \\
\hline Gal-1 tumor cell & & & & & & & & & & & & \\
\hline Weak & 24 & $34.3 \%$ & 15 & $62.5 \%$ & 9 & $37.5 \%$ & $001+$ & & & & & \\
\hline Strong & 46 & $65.7 \%$ & 7 & $15.2 \%$ & 39 & $84.8 \%$ & $-0.001+$ & & & & & \\
\hline
\end{tabular}

Continuous variables were expressed as mean $\pm \mathrm{SD} \&$ median (range); Categorical variables were expressed as number (percentage); $\bullet$ Mann Whitney $\mathrm{U}$ test; $\ddagger$ Chi-square test; $§$ Chi-square test for trend; $\mathrm{p}<0.05$ is significant.

Comparing 1-year, 2 years and 3 years OS rates, it was found to be significantly higher regarding the absence of lymphadenopathy $(\mathrm{p}<0.001)$ and FIGO stage $(\mathrm{p}=0.019)$

Gal-1 expression in peritumoral stromal cells was also significant $(p=0.002)$, but no statistically significant difference was found regarding Gal-1 tumor cell expression ( $\mathrm{p}=0.064)$ (Table 7, Table 8, Figure 2). 


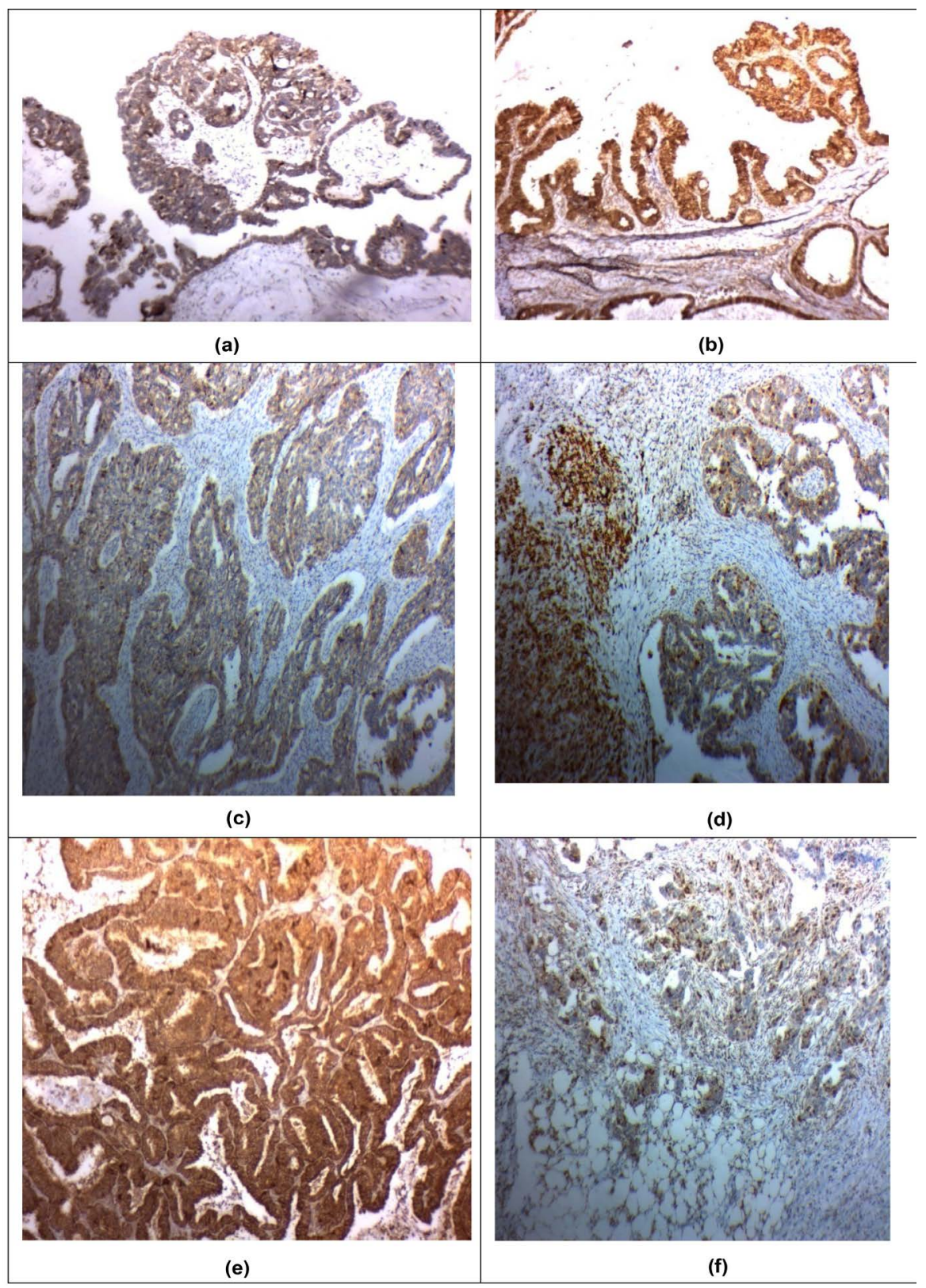

Figure 1. Immunohistochemical expression of Galectin-1: (a) Low grade SOC showing weak Gal-1 IHC expression ( $\times 200)$; (b) Low grade SOC showing strong Gal-1 IHC expression (×200); (c) High grade SOC showing weak Gal-1 IHC expression (×200); (d) High grade SOC showing weak Gal-1 tumor IHC expression but strong stromal expression (×200); (e) High grade SOC showing strong Gal-1 IHC expression (×200); (f) Metastatic deposit in omentum from stage IV patient showing Gal-1 tumor and stromal weak expression $(\times 200)$.

\section{Discussion}

Epithelial ovarian cancers (EOC) represent $90 \%$ of ovarian cancers which is the most common cause of death from gynecological malignancies and there has been a sustained interest to identify new biomarkers that detect progression and prognosis of EOC [2]. 
Table 7. Relation between clinicopathological features, immunohistochemical staining and overall survival among 70 ovarian carcinoma patients.

\begin{tabular}{|c|c|c|c|c|c|c|c|}
\hline & \multirow{2}{*}{$\mathrm{N}$} & \multicolumn{2}{|c|}{ Mean OS (months) } & \multicolumn{3}{|c|}{ Overall Survival (OS) } & \multirow{2}{*}{ p-value $\dagger$} \\
\hline & & Estimate & (95\% CI) & 1-year & 2-year & 3 -year & \\
\hline All patients & 70 & 28.22 month & $(24.92-31.52)$ & $83.2 \%$ & $67.3 \%$ & $67.3 \%$ & ---- \\
\hline \multicolumn{8}{|l|}{ Grade } \\
\hline Low & 18 & 32.09 month & $(28.38-35.80)$ & $93.8 \%$ & $82 \%$ & $82 \%$ & 0.063 \\
\hline High & 52 & 26.51 month & $(22.34-30.68)$ & $79.5 \%$ & $63.1 \%$ & $63.1 \%$ & \\
\hline \multicolumn{8}{|l|}{$\underline{\mathrm{LN}}$} \\
\hline Negative & 22 & 35.00 month & $(33.17-36.88)$ & $100 \%$ & $92.3 \%$ & $92.3 \%$ & $<0.001$ \\
\hline Positive & 48 & 16.07 month & $(14.10-18.03)$ & $74.6 \%$ & ----- & ----- & \\
\hline \multicolumn{8}{|l|}{ Stage } \\
\hline Stage I & 8 & 36.00 month & ----- & $100 \%$ & $100 \%$ & $100 \%$ & 0.019 \\
\hline Stage II & 12 & 30.97 month & $(27.20-34.75)$ & $100 \%$ & $78.6 \%$ & ----- & \\
\hline Stage III & 32 & 17.05 month & $(14.79-19.32)$ & $79.3 \%$ & ----- & ---- & \\
\hline Stage IV & 18 & 12.69 month & $(10.47-14.90)$ & $68.8 \%$ & ----- & ----- & \\
\hline \multicolumn{8}{|l|}{ Gal-1 } \\
\hline Weak & 22 & 35.00 month & ---- & $100 \%$ & $100 \%$ & $100 \%$ & 0.002 \\
\hline Strong & 48 & 24.22 month & $(19.67-28.77)$ & $75.5 \%$ & $49.5 \%$ & $49.5 \%$ & \\
\hline \multicolumn{8}{|c|}{ Gal-1 tumor cells } \\
\hline Weak & 24 & 31.60 month & $(28.02-35.19)$ & $87.1 \%$ & $87.1 \%$ & $87.1 \%$ & 0.064 \\
\hline Strong & 46 & 25.10 month & $(20.33-29.87)$ & $81.2 \%$ & $51.9 \%$ & $51.9 \%$ & \\
\hline
\end{tabular}

Continuous variables were expressed as mean $(95 \% \mathrm{CI})$; categorical variables were expressed as number (percentage); $†$ Log rank test; $\mathrm{p}<0.05$ is significant.

CA125 is the most clinically useful ovarian cancer biomarker, but it is not secreted by $20 \%$ of EOC. Also, it lacks both sensitivity and specificity in early detection, thus identification of more specific and sensitive markers to detect patients at early stages is required [18].

This need for a new marker is essential because of high rate of recurrence of ECO after treatment [19]. The poor survival rate is due to high grade serous carcinomas and its late diagnosis [20].

Galectin-1 is a $14-\mathrm{kDa}$ laminin-binding galectin, that may have a role in a variety of physiological and pathological processes as cell-cell and cell matrix interaction, cell growth, inflammatory and immune regulation [21].

Galectin-1 was detected to accumulate in ovarian cancers. However, its potential as diagnostic and prognostic marker for serous ovarian carcinoma is unclear [15].

The aim of this study was to evaluate circulating galectin-1 level as well as its expression by immunohistochemical staining in tumor and peri-tumoral stromal cells in SOC trying to find its significance in diagnosis and prognosis of these tumors. 
Table 8. Relation between clinicopathological features, immunohistochemical staining and mortality among 70 ovarian carcinoma patients.

\begin{tabular}{|c|c|c|c|c|c|c|c|}
\hline \multirow[t]{2}{*}{ Characteristics } & \multicolumn{2}{|c|}{$\begin{array}{c}\text { All } \\
(\mathrm{N}=70)\end{array}$} & \multicolumn{2}{|c|}{$\begin{array}{c}\text { Alive } \\
(\mathrm{N}=54)\end{array}$} & \multicolumn{2}{|c|}{$\begin{array}{c}\text { Died } \\
(\mathrm{N}=16)\end{array}$} & \multirow[t]{2}{*}{ p-value } \\
\hline & No. & $\%$ & No. & $\%$ & No. & $\%$ & \\
\hline \multicolumn{8}{|l|}{ Age (years) } \\
\hline Mean \pm SD & \multicolumn{2}{|c|}{$50.24 \pm 9.58$} & \multicolumn{2}{|c|}{$49.92 \pm 9.57$} & \multicolumn{2}{|c|}{$51.31 \pm 9.85$} & \\
\hline $\begin{array}{l}\text { Median } \\
\text { (Range) }\end{array}$ & \multicolumn{2}{|c|}{$\begin{array}{c}52.50 \\
(27-62)\end{array}$} & \multicolumn{2}{|c|}{$\begin{array}{c}52 \\
(27-62)\end{array}$} & \multicolumn{2}{|c|}{$\begin{array}{c}54 \\
(28-61)\end{array}$} & $0.524^{\bullet}$ \\
\hline \multicolumn{8}{|l|}{$\underline{\text { Grade }}$} \\
\hline $\begin{array}{l}\text { Low } \\
\text { High }\end{array}$ & $\begin{array}{l}18 \\
52\end{array}$ & $\begin{array}{l}27.7 \% \\
74.3 \%\end{array}$ & $\begin{array}{l}16 \\
38\end{array}$ & $\begin{array}{l}88.9 \% \\
73.1 \%\end{array}$ & 2 & $\begin{array}{l}11.1 \% \\
26.9 \%\end{array}$ & $0.209 \ddagger$ \\
\hline \multicolumn{8}{|l|}{$\underline{\mathrm{LN}}$} \\
\hline $\begin{array}{l}\text { Negative } \\
\text { Positive }\end{array}$ & $\begin{array}{l}22 \\
48\end{array}$ & $\begin{array}{l}31.4 \% \\
68.6 \%\end{array}$ & $\begin{array}{l}21 \\
33\end{array}$ & $\begin{array}{l}95.5 \% \\
68.8 \%\end{array}$ & $\begin{array}{c}1 \\
15\end{array}$ & $\begin{array}{r}4.5 \% \\
31.2 \%\end{array}$ & $0.014 \ddagger$ \\
\hline \multicolumn{8}{|l|}{$\underline{\text { Stage }}$} \\
\hline Stage I & 8 & $11.4 \%$ & 8 & $100 \%$ & 0 & $0 \%$ & \multirow{4}{*}{$0.056 \S$} \\
\hline Stage II & 12 & $17.1 \%$ & 10 & $83.3 \%$ & 2 & $16.7 \%$ & \\
\hline Stage III & 32 & $45.7 \%$ & 24 & $75 \%$ & 8 & $25 \%$ & \\
\hline Stage IV & 18 & $25.7 \%$ & 12 & $66.7 \%$ & 6 & $33.3 \%$ & \\
\hline \multicolumn{8}{|l|}{$\underline{\mathrm{CA} 125(\mathrm{u} / \mathrm{ml})}$} \\
\hline Mean \pm SD & \multicolumn{2}{|c|}{$1071.31 \pm 666.91$} & \multicolumn{2}{|c|}{$960.01 \pm 670.88$} & \multicolumn{2}{|c|}{$1446.93 \pm 510.49$} & \\
\hline $\begin{array}{l}\text { Median } \\
\text { (Range) }\end{array}$ & \multicolumn{2}{|c|}{$\begin{array}{c}1238.50 \\
(69-2008)\end{array}$} & \multicolumn{2}{|c|}{$\begin{array}{c}1233.50 \\
(69-2005)\end{array}$} & \multicolumn{2}{|c|}{$\begin{array}{c}1253.50 \\
(90-2008)\end{array}$} & $0.009 \bullet$ \\
\hline \multicolumn{8}{|l|}{$\underline{\text { Gal-1 (ng/ml) }}$} \\
\hline Mean \pm SD & \multicolumn{2}{|c|}{$334.84 \pm 187.67$} & \multicolumn{2}{|c|}{$296.25 \pm 174.70$} & \multicolumn{2}{|c|}{$465.05 \pm 175.31$} & \\
\hline $\begin{array}{l}\text { Median } \\
\text { (Range) }\end{array}$ & \multicolumn{2}{|c|}{$\begin{array}{c}277 \\
(32-763)\end{array}$} & \multicolumn{2}{|c|}{$\begin{array}{c}271 \\
(32-649)\end{array}$} & \multicolumn{2}{|c|}{$\begin{array}{c}380 \\
(280-763)\end{array}$} & $<0.001^{\bullet}$ \\
\hline$\underline{\text { Gal-1 }}$ & & & & & & & \\
\hline Weak & 22 & $31.4 \%$ & 22 & $100 \%$ & 0 & $0 \%$ & \\
\hline Strong & 48 & $68.6 \%$ & 32 & $66.7 \%$ & 16 & $33.3 \%$ & $0.002+$ \\
\hline Gal-1 tumor cells & & & & & & & \\
\hline Weak & 24 & $34.3 \%$ & 21 & $87.5 \%$ & 3 & $12.5 \%$ & $0.136 \ddagger$ \\
\hline Strong & 46 & $65.7 \%$ & 33 & $71.7 \%$ & 13 & $28.3 \%$ & \\
\hline
\end{tabular}

Continuous variables were expressed as mean \pm SD \& median (range); Categorical variables were expressed as number (percentage); •Mann Whitney $\mathrm{U}$ test; $¥$ Chi-square test; $§$ Chi-square test for trend; $\mathrm{p}<0.05$ is significant.

We studied serum level of Gal-1 in 70 patients with SOC at different stages and 25 healthy controls. The mean values of serum Gal-1 were significantly higher in SOC patients than in controls $(\mathrm{p}<0.001)$ 


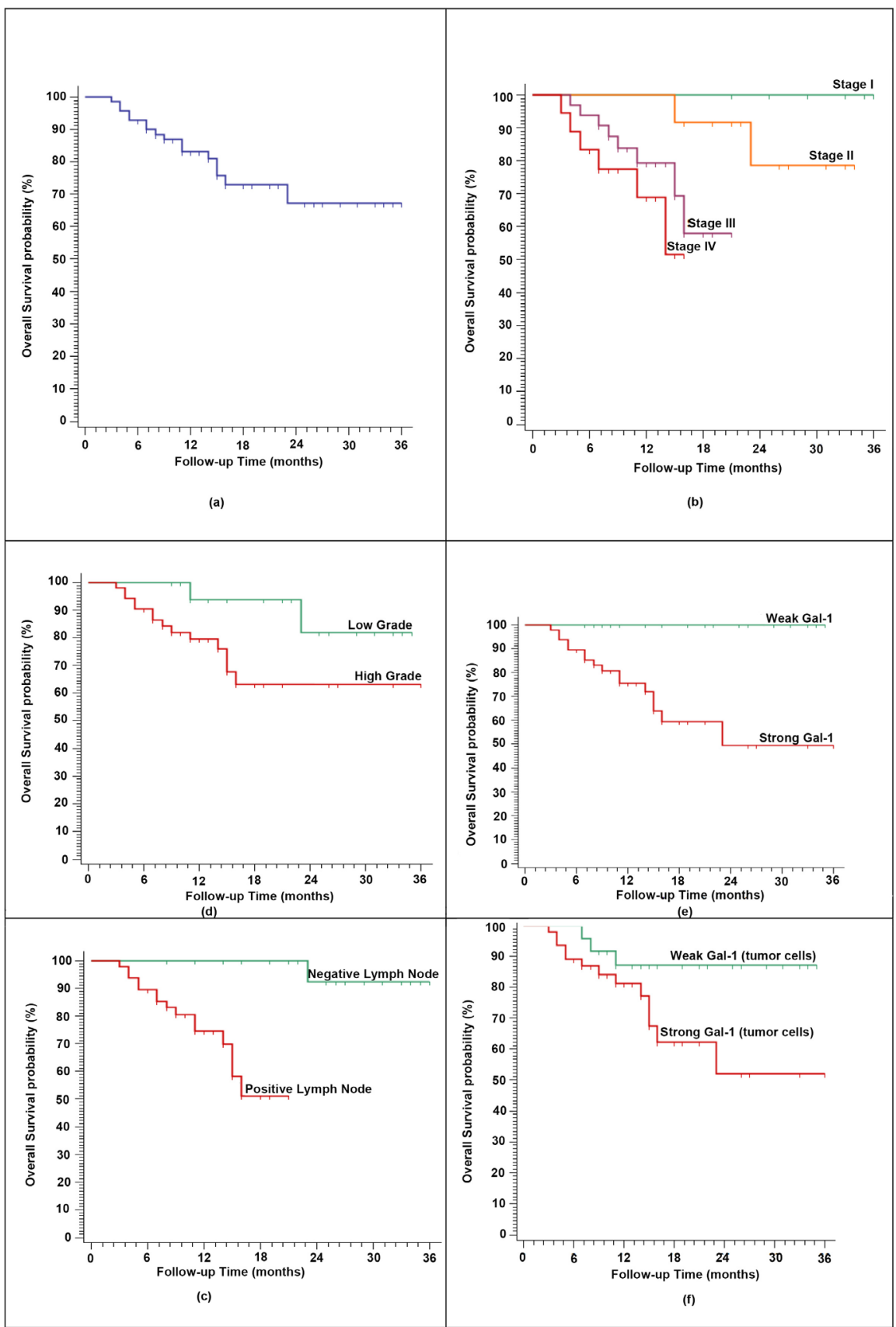

Figure 2. Kaplan Meier plot of Overall Survival, (a) All studied patients; (b) Stratified by grade; (c) Stratified by LN spread; (d) Stratified by FIGO stage; (e) Stratified by Gal-1 and (f) Stratified by Gal-1 in tumor cells.

Serum level of Gal-1 was significantly associated with the FIGO stage of SOC ( $\mathrm{p}<0.001)$ and higher in stage III, IV compared to stage 1, II. So normal or low Gal-1 level ; may suggest absence of metastasis.

These results are supported by Chen et al., 2015 [17] who reported that serum Gal-1 level was elevated in relation to tumor progression, and they suggested that the increase in serum Gal-1 level is associated with the occurrence of metastasis. 
So, we can imply that the metastatic spread of SOC is associated with higher level of circulating Gal-1. This finding may open a view for detection of early spread of SOC.

Serum level of CA125 was significantly raised in patients with SCO compared to controls $\mathrm{p}<00.001$, a finding that is supported by Hogdal et al., 2007 [22].

We reported a statistically significant direct correlation between serum Gal-1 level and CA 125 level in SOC, a finding that is supported by Labrie et al., 2017 [6]. On comparing both of them in diagnosis of SOC we found that serum Gal-1 was more accurate than does CA125, as serum Gal-1 was $100 \%$ specific, sensitive and accurate in diagnosis of SCO at cut off value of $135 \mathrm{ng} / \mathrm{ml}$ with positive and negative predictive values of $100 \%$, while CA 125 at cut off value $>49 \mathrm{u} / \mathrm{ml}$ was $88.5 \% 96 \%, 98.4 \%, 75 \%$ and $90.5 \%$ in sensitivity, specificity, positive, negative predictive values and accuracy in diagnosis of SOC $\mathrm{p}<0.001$.

Similar results were obtained by Chen et al., 2015 [17] who found that the association between Gal-1 and CA125 is highly significant, that confirm the lack of independence between these two markers. They found also Gal-1 was more sensitive than CA125 as a false positive obtained by CA125 was negative obtained by Gal-1, and $98 \%$ of SOC who are positive by CA 125 were positive by Gal-1.

From these results we can suggest measuring Gal-1 in every patient diagnosed with SOC to simplify decision making before treatment as high serum level suggest metastasis.

After immunohistochemical staining of the 70 cases, we found that strong expression of Gal-1 was detected in tumor cells in 46 patients (65.7\%) which was much higher than patients with weak expression (34.3\%). We also found strong expression in peri-tumoral stroma in 48 patients (68.6\%), a percent which is higher than that with weak expression $\mathrm{p}<0.001$

High serum level of Gal-1 in SOC patients was associated with strong Gal-1 expression in tumor and stromal cells compared to those with weak expression $\mathrm{p}$ $<0.001$ and $\mathrm{p}=0.002$, respectively.

Similar results were obtained for serum level of CA125 and expression of Gal-1 in tumors and peri-tumor stromal cells, the level was higher in Gal-1 strong expression cases compared to weak expression $\mathrm{p}=0.001$ and $\mathrm{p}=0.025$ respectively.

After immunohistochemical staining, tumor cell strong expression of Gal-1 was more frequent in patients with advanced stage compared with early stages as it was found in $37.5 \%, 50 \%, 68.8 \%$ and $83.3 \%$ in stage I, II, III, IV respectively.

Strong Gal-1 expression in stromal cells was also more frequent in advanced stages of SOC (25\%, 66.7\%, 68.8\% and $88.9 \%)$ in stages I, II, III, IV respectively. Labrie et al., 2017 found strong association between Gal-1 expression and higher FIGO stage of SOC.

Gal-1 showed strong IHC expression both in tumor and peri-tumoral stroma cells in the presence of lymphadenopathy, but did not reach statistically significant level. 
High grade SOC were associated with strong Gal-1 expression both in tumor and stromal cells compared to low grade ( $84.6 \%$ vs $11.1 \%) \mathrm{p}<0.001$ and $982.7 \%$ vs $27.8 \%) \mathrm{p}<0.001$, respectively.

There was a significantly statistic difference between survivors and non-survivors as regarding Gal-1 expression in peritumoral stromal cells $(\mathrm{p}=0.002)$. Gal-1 expression is associated with poor survival. This was in accordance with Chen et al., 2015 [17] and Labrie et al., 2017 who found that strong Gal-1 expression in tumor cells and stromal cells is associated with poor prognosis. No significant difference was detected between survivors and non-survivors regarding Gal-1 expression in tumor cells $\mathrm{p}=0.136$, a result supported by Chen et al., 2015 [17] and Labrie et al., 2017 [6].

High serum level of both Gal-1 and CA125 were significantly associated with poor survival $\mathrm{p}<0.001$ and $\mathrm{p}=0.009$ respectively as supported by Labrie et al., 2017 and they stated that high Gal-1 in circulation and its tissue expression in SOC may be useful for follow up patients with SOC.

This poor prognosis of SOC with high expression of Gal-1 in circulation and in peritumoral stromal cells suggests its possible role in favoring metastasis through enhancing spread of tumor cells, promoting their embolization, elevating tumor vascular permeability and conferring a selective support to metastatic cells [23] [24].

These results that demonstrated the increased Gal-1 in serum and cancer associated stromal cells could be important in cancer progression and poor survival.

After follow up period of 3 years with the mean of 14 months, we found overall survival rate was associated with strong stromal Gal-1 expression $\mathrm{p}=0.002$, stage of SOC, $\mathrm{p}=0.009$ and the presence of lymphatic spread $\mathrm{p}<0.001$.

These results support the usefulness of Gal-1 immunohistochemical expression in peri-tumoral cells as prognostic biomarker for the possibility of successful treatment or the possibility of chemotherapeutic resistance.

These findings were supported by Chen et al., 2015 [17] and Labrie et al., 2017 [6] who found 5-years OS and DFS were associated with strong Gal-1 expression in peri-tumoral stromal cells.

There may be some possible limitations of our study as the limited number of patients included the financial problems and the difficulty of follow up. We recommend a study on large scale.

\section{Conclusion}

We can conclude that serum Gal-1 and its tissue level are over-expressed in SOC patients on progression of disease; this may support its usefulness as non-invasive biomarker for diagnosis and prognosis of these patients.

\section{Author's Contributions}

All authors contributed in this research paper and have approved the final article. 


\section{Funding}

There are no funding sources for this research. It was completely paid by the researchers with no external funding sources.

\section{Conflicts of Interest}

The authors have declared no conflict of interest.

\section{References}

[1] Bodurka, D.C., Deavers, M.T., Tian, C., Sun, C.C., Malpica, A., Coleman, R.L., et al. (2012) Reclassification of Serous Ovarian Carcinoma by a 2-Tier System: A Gynecologic Oncology Group Study. Cancer, 118, 3087-3094.

https://doi.org/10.1002/cncr.26618

[2] Siegel, R.L., Miller, K.D. and Jemal, A. (2016) Cancer Statistics 2016. CA: A Cancer Journal for Clinicians, 66, 7-30. https://doi.org/10.3322/caac.21332

[3] Davidson, B. and Trope, C.G. (2014) Ovarian Cancer: Diagnostic, Biological and Prognostic Aspects. Women's Health, 10, 519-533.

https://doi.org/10.2217/WHE.14.37

[4] Baldwin, L.A., Huang, B., Miller, R.W., Tucker, T., Goodrich, S.T., Podzielinski, I., DeSimone, C.P., Ueland, F.R., van Nagell, J.R. and Seamon, L.G. (2012) Ten-Year Relative Survival for Epithelial Ovarian Cancer. Obstetrics \& Gynecology, 120, 612-618. https://doi.org/10.1097/AOG.0b013e318264f794

[5] Heublein, S., Sabina, K.P., Doris, M., Nina, D. and Udo, J. (2016) p53 Determines Prognostic Significance of the Carbohydrate Stem Cell Marker TF1 (CD176) in Ovarian Cancer. Journal of Cancer Research and Clinical Oncology, 142, 1163-1170. https://doi.org/10.1007/s00432-016-2126-3

[6] Labrie, M., Oliveira, L., Araujo, F., Communal, L., Mes-Masson, A. and St-Pierre, Y. (2017) Tissue and Plasma Levels of Galectins in Patients with High Grade Serous Ovarian Carcinoma as New Predictive Biomarkers. Scientific Reports, 7, Article No. 13244. https://doi.org/10.1038/s41598-017-13802-5

[7] Vaughan, S., Coward, J.I., Bast Jr., R.C., Berchuck, A., Berek, J.S., Brenton, J.D., et al. (2011) Rethinking Ovarian Cancer: Recommendations for Improving Outcomes. Nature Reviews Cancer, 11, 719-725. https://doi.org/10.1038/nrc3144

[8] Astorgues-Xerri, L., Riveiro, M.E., Tijeras-Raballand, A., et al. (2014) Unraveling Galectin-1 as a Novel Therapeutic Target for Cancer. Cancer Treatment Reviews, 40, 307-319. https://doi.org/10.1016/j.ctrv.2013.07.007

[9] Cousin, J.M. and Cloninger, M.J. (2016) The Role of Galectin-1 in Cancer Progression, and Synthetic Multivalent Systems for the Study of Galectin-1. International Journal of Molecular Sciences, 17, 1566. https://doi.org/10.3390/ijms17091566

[10] Kovacs-Solyom, F., Blasko, A., Fajka-Boja, R., Katona, R.L., Vegh, L., Novak, J., et al. (2010) Mechanism of Tumor Cell-Induced T-Cell Apoptosis Mediated by Galectin-1. Immunology Letters, 127, 108-118.

https://doi.org/10.1016/j.imlet.2009.10.003

[11] Chiariotti, L., Berlingieri, M.T., Battaglia, C., Benvenuto, G., Martelli, M.L., Salvatore, P., et al. (1995) Expression of Galectin-1 in Normal Human Thyroid Gland and in Differentiated and Poorly Differentiated Thyroid Tumors. International Journal of Cancer, 64, 171-175. https://doi.org/10.1002/ijc.2910640305

[12] Xu, X.C., el-Naggar, A.K. and Lotan, R. (1995) Differential Expression of Galectin-1 
and Galectin-3 in Thyroid Tumors. Potential Diagnostic Implications. The American Journal of Pathology, 147, 815-822.

[13] Jung, E.J., Moon, H.G., Cho, B.I., Jeong, C.Y., Joo, Y.T., Lee, Y.J., et al. (2007) Galectin-1 Expression in Cancer-Associated Stromal Cells Correlates Tumor Invasiveness and Tumor Progression in Breast Cancer. International Journal of Cancer, 120, 2331-2338. https://doi.org/10.1002/ijc.22434

[14] Van den Brule, F.A., Waltregny, D. and Castronovo, V. (2001) Increased Expression of Galectin-1 in Carcinoma-Associated Stroma Predicts Poor Outcome in Prostate Carcinoma Patients. The Journal of Pathology, 193, 80-87. https://doi.org/10.1002/1096-9896(2000)9999:9999<::AID-PATH730>3.0.CO;2-2

[15] Zhang, P., Zhang, P., Shi, B., Zhou, M., Jiang, H., Zhang, H., et al. (2014) Galectin-1 Overexpression Promotes Progression and Chemoresistance to Cisplatin in Epithelial Ovarian Cancer. Cell Death \& Disease, 5, e991. https://doi.org/10.1038/cddis.2013.526

[16] Vertongen, F., Toubeau, G., Decaestecker, C. and Kiss, R. (2008) The Determination of the Levels of Circulating Galectin-1 and -3 in HNSCC Patients Could Be Used to Monitor Tumor Progression and or Responses to Therapy. Oral Oncology, 44, 86-93. https://doi.org/10.1016/j.oraloncology.2006.12.014

[17] Chen, L., Yao, Y., Sun, L., et al. (2015) Clinical Implication of the Serum Galectin-1 Expression in Epithelial Ovarian Cancer Patients. Journal of Ovarian Research, 8, 78. https://doi.org/10.1186/s13048-015-0206-7

[18] Badgwell, D. and Bast Jr., R.C. (2007) Early Detection of Ovarian Cancer. Disease Markers, 23, 397-410. https://doi.org/10.1155/2007/309382

[19] Box, C., Rogers, S.J., Mendiola, M. and Eccles, S.A. (2010) Tumor Micro-Environmental Interactions: Paths to Progression and Targets for Treatment. Seminars in Cancer Biology, 20, 128-138. https://doi.org/10.1016/j.semcancer.2010.06.004

[20] Turcotte, M., Spring, K., Pommey, S., Chouinard, G., Cousineau, I., George, J., et al. (2015) CD73 Is Associated with Poor Prognosis in High-Grade Serous Ovarian Cancer. Cancer Research, 75, 4494-4503. https://doi.org/10.1158/0008-5472.CAN-14-3569

[21] Camby, I., Le, M.M., Lefranc, F. and Kiss, R. (2006) Galectin-1: A Small Protein with Major Functions. Glycobiology, 16, 137R-157R. https://doi.org/10.1093/glycob/cwl025

[22] Hogdall, E.V., Christensen, L., Kjaer, S.K., Blaakaer, J., Kjaerbye-Thygesen, A., Gayther, S., et al. (2007) CA125 Expression Pattern, Prognosis and Correlation with Serum CA125 in Ovarian Tumor Patients. From the Danish "MALOVA" Ovarian Cancer Study. Gynecologic Oncology, 104, 508-515.

https://doi.org/10.1016/j.ygyno.2006.09.028

[23] Suzuki, O. and Abe, M. (2014) Galectin-1-Mediated Cell Adhesion, Invasion and Cell Death in Human Anaplastic Large Cell Lymphoma: Regulatory Roles of Cell Surface Glycans. International Journal of Oncology, 44, 1433-1442. https://doi.org/10.3892/ijo.2014.2319

[24] Wu, M.H., Ying, N.W., Hong, T.M., Chiang, W.F., Lin, Y.T. and Chen, Y.L. (2014) Galectin-1 Induces Vascular Permeability through the Neuropilin-1/Vascular Endothelial Growth Factor Receptor-1 Complex. Angiogenesis, 17, 839-849. https://doi.org/10.1007/s10456-014-9431-8 